\title{
The Role of Artificial Intelligence in Cardiovascular Imaging: State of the Art Review
}

\author{
Karthik Seetharam, Daniel Brito, Peter D. Farjo and Partho P. Sengupta* \\ Department of Cardiology, West Virginia University Medicine Heart \& Vascular Institute, Morgantown, WV, United States
}

In this current digital landscape, artificial intelligence (Al) has established itself as a powerful tool in the commercial industry and is an evolving technology in healthcare. Cutting-edge imaging modalities outputting multi-dimensional data are becoming increasingly complex. In this era of data explosion, the field of cardiovascular imaging is undergoing a paradigm shift toward machine learning $(\mathrm{ML})$ driven platforms. These diverse algorithms can seamlessly analyze information and automate a range of tasks. In this review article, we explore the role of $\mathrm{ML}$ in the field of cardiovascular imaging.

Keywords: machine learning, automation, cardiovascular imaging, precision medicine, advanced imaging

OPEN ACCESS

Edited by:

Salah D. Qanadli,

University of Lausanne, Switzerland

Reviewed by:

Giulia Elena Mandoli,

University of Siena, Italy

PierGiorgio Masci,

King's College London,

United Kingdom

*Correspondence:

Partho P. Sengupta

partho.sengupta@wvumedicine.org

Specialty section:

This article was submitted to

Cardiovascular Imaging

a section of the journal

Frontiers in Cardiovascular Medicine

Received: 18 October 2020 Accepted: 08 December 2020 Published: 23 December 2020

Citation:

Seetharam K, Brito D, Farjo PD and Sengupta PP (2020) The Role of Artificial Intelligence in Cardiovascular Imaging: State of the Art Review. Front. Cardiovasc. Med. 7:618849,

doi: 10.3389/fcvm.2020.618849

\section{INTRODUCTION}

As technology continues to evolve at a rapid pace, each and every significant innovation has a phenomenal capability of transforming various aspects of society $(1,2)$. In this era of miniaturized devices and smartphone applications, continuous streams of individualized information have become the standard of life $(3,4)$. Similarly, the adoption of artificial intelligence (AI) in medicine has far-reaching potential, especially in the field of cardiovascular imaging $(2,5,6)$. With a vast array of imaging modalities at our disposal, these approaches provide limitless information regarding cardiac structure and function (7). In parallel to the technological revolution, imaging approaches also continue to grow significantly $(5,8)$. Novel parameters are added to existing techniques, providing additional information regarding the cardiac function $(7,9,10)$. However, are more data points beneficial if they cannot be used in routine clinical practice? (11). Information necessary to medical management needs to be prioritized first rather than having a cacophony of data points.

The utility of AI in cardiovascular imaging bridges the gap between new technology, big data, and the clinical provider (12-14). Machine learning (ML), a branch of AI, is especially pertinent in cardiovascular imaging as it can analyze large amounts of information in a multitude of approaches $(1,15,16)$. ML can connect information from a variety of interfaces and present it in a meaningful manner for the practitioner $(13,14)$. Also, it can automate several measurements in various imaging modalities $(17,18)$. The growth of AI will facilitate the progression of precision medicine. In this review article, we assess the role of AI and ML in cardiovascular imaging.

\section{EVOLUTION OF BIG DATA}

Imaging modalities permit the visual assessment of cardiac function and detect underlying cardiac pathology. A single scan produces an abundance of clinical and operational data (19). For example, a single echocardiogram can produce roughly 2 gigabytes of data. Since millions of patients undergo echocardiography annually, this translates to petabytes of information being collected. With the emergence of higher processing capabilities of computer processing units (CPU) and 
cloud infrastructures, the newer system can process a plethora of data in real-time $(5,20)$. Imaging data can be quite complex and present in varying dimensions (two or three or 4 dimensions) and formats which include digital imaging and communications in medicine (DICOM), moving picture experts Group (MPEG), and joint photographic experts group (JPEG) (19). This results in exceedingly high dimensionality of data and predisposes to significant difficulties in clinical practice (21).

Big data is heavily utilized in clinical research (22). Many academic centers invest vast resources in generating and large data sets for various research endeavors. The findings from large data sets are generally applicable to vast portions of the population. They provide more consistent and reliable findings than smaller or single institutional studies. In the near future, big data will incorporate genetic or molecular parameters for the patient or pan-omic data sets $(22,23)$.

\section{EMERGING SIGNIFICANCE OF AI}

As stated earlier, data is becoming increasingly complex with rapidly advancing changes in technology (18). Big data with countless, non-linear associations will exceed the capabilities of existing frequentist or Bayesian statistical approaches $(23,24)$. Although they are the current gold standard in current research, this may not hold true in the foreseeable future.

In contrast, $\mathrm{ML}$ and $\mathrm{AI}$ are far more dynamic in nature (14). With this transition to big data, ML algorithms will play a pivotal role in the days to come. As the size and complexity of data increases, the performance of ML increases proportionally $(5,25)$. ML frameworks can further expand our knowledge regarding different cardiac pathology. It can connect information from a variety of different interfaces.

\section{TYPES OF MACHINE LEARNING}

ML is an umbrella term that refers to a collection of various analytical algorithms [(5); Table 1]. It can be broadly classified into supervised learning, unsupervised learning, semisupervised learning, and reinforcement learning (26). Among these, supervised and unsupervised learning are frequently used in clinical research (27). Supervised learning operates within domains of labels or annotations within a dataset $(18,28)$. Whereas, unsupervised learning looks purely at data points independent form labels and is considered agnostic $(29,30)$. Semi-supervised learning contains properties of both supervised and unsupervised learning (12). The last among these ML frameworks is reinforcement learning. Reinforcement learning is similar to human psychology, utilizes certain reward criteria for the algorithm to perform functions within a dataset (22). It is yet to gain significant traction in the field of cardiovascular imaging.

\section{RISING RELEVANCE OF DEEP LEARNING}

Among ML algorithms, deep learning has the most potential in innovation and discovery $(24,31)$. It is becoming increasingly popular due to significant strides in cloud infrastructures and
TABLE 1 | A table describing different types of machine learning with various examples.

\begin{tabular}{|c|c|c|}
\hline $\begin{array}{l}\text { Types of machine } \\
\text { learning }\end{array}$ & Function & Examples \\
\hline $\begin{array}{l}\text { Supervised learning } \\
\text { (12) }\end{array}$ & $\begin{array}{l}\text { The dataset has labels and } \\
\text { outcomes, infers from data } \\
\text { for prediction purposes }\end{array}$ & $\begin{array}{l}\text { Encompasses logistic } \\
\text { regression, ridge regression, } \\
\text { elastic net regression, } \\
\text { Bayesian network, artificial } \\
\text { neural network }\end{array}$ \\
\hline $\begin{array}{l}\text { Unsupervised } \\
\text { learning (12) }\end{array}$ & $\begin{array}{l}\text { The dataset contains no } \\
\text { labels, detects pivotal } \\
\text { relationships }\end{array}$ & $\begin{array}{l}\text { This contains hierarchical } \\
\text { clustering, k-means } \\
\text { clustering, principal } \\
\text { component analysis }\end{array}$ \\
\hline $\begin{array}{l}\text { Semi-supervised } \\
\text { learning (12) }\end{array}$ & $\begin{array}{l}\text { A combination of supervised } \\
\text { and unsupervised learning }\end{array}$ & $\begin{array}{l}\text { Frequently used in image } \\
\text { and speech recognition }\end{array}$ \\
\hline $\begin{array}{l}\text { Re-enforcement } \\
\text { learning (12) }\end{array}$ & $\begin{array}{l}\text { Utilizes reward function to } \\
\text { execute tasks }\end{array}$ & $\begin{array}{l}\text { Commonly seen in medical } \\
\text { imaging, analytics, and } \\
\text { prescription selection }\end{array}$ \\
\hline
\end{tabular}

augmented computing prowess (12). Deep learning is the foundation for cutting-edge technology like voice recognition software such as Siri or Alex and self-driving cars $(1,32)$. In comparison to other ML frameworks, deep learning performs significantly better with larger and complex datasets. The architecture of deep learning is similar to the neuronal structure present within humans $(33,34)$. Arranged in a series of layers, information is processed from preceding and successive layers in an intricate manner to extrapolate outcomes present within vast data matrices (25). Other algorithms require significant training to obtain satisfactory results. However, the accuracy of deep learning can be easily improved by elevating the network capacity or augmenting the training dataset. It requires less domain knowledge to execute a function.

There are also several subtypes present in deep learning. One of the most commonly used deep learning frameworks is a convolutional neural network (CNN) (31). It contains a convolutional component responsible for feature extraction and has a fully connected enabling classification. In fully connected networks (FNN), every unit in any layer is linked to every unit in prior and succeeding layers (31). Recurrent neural networks (RNN) employ feedback loops to comprehend various inputs (31).

\section{ROLE OF AI IN ECHOCARDIOGRAPHY}

Echocardiography is the most widely used imaging technique in cardiac care (10). It plays an indispensable role in assessing cardiac function and it identifies various cardiac pathology (10). Over the last 33 years, significant progression in echocardiographic automated quantification has occurred (Figure 1).

ML algorithms can make new echocardiographic technology clinically relevant. With the emergence of cardiac strain, it can evaluate the cardiac function beyond the ejection fraction. Ejection fraction is hailed as the gold standard metric for cardiac assessment. Although strain can fundamentally alter clinical care, 


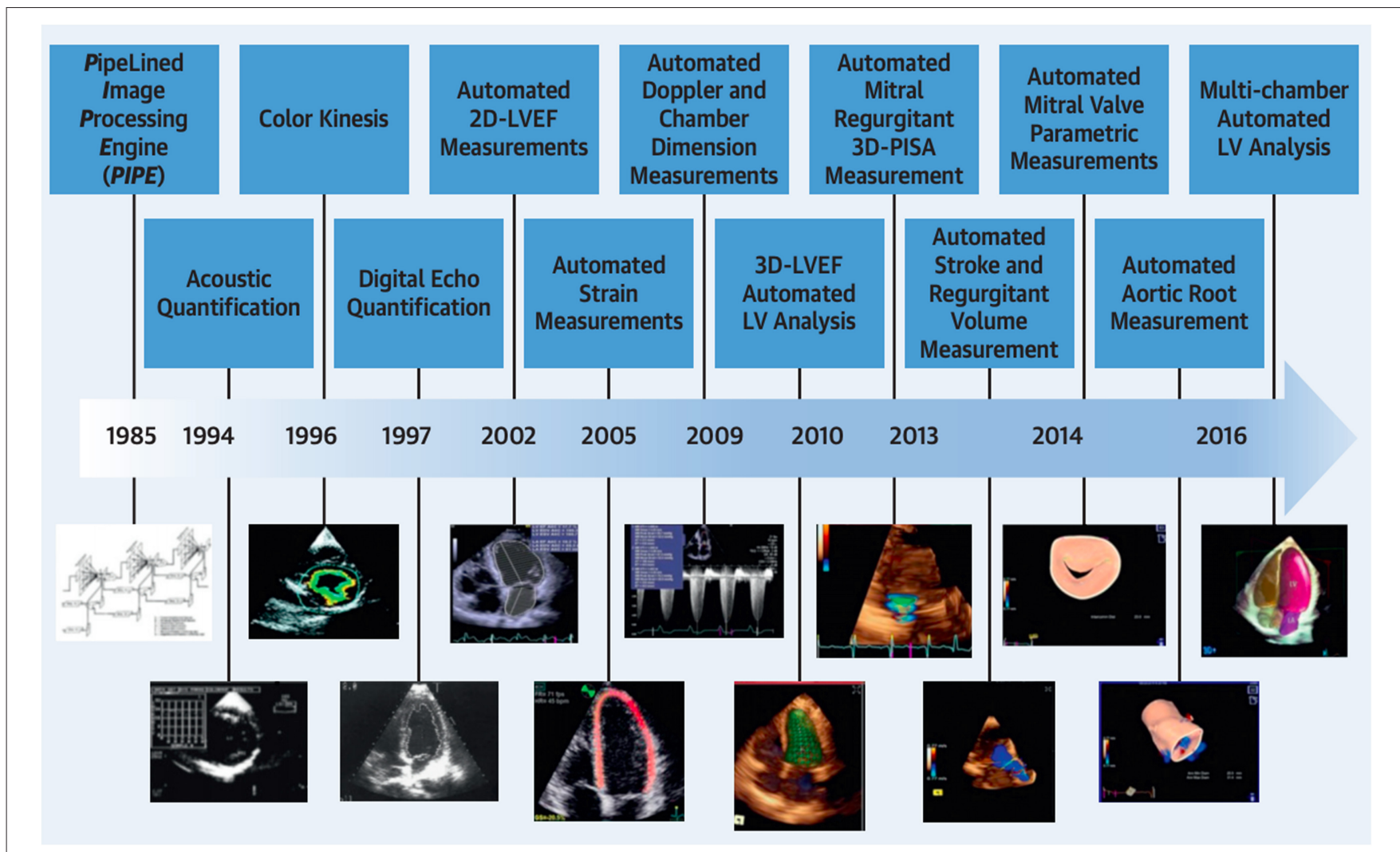

FIGURE 1 | Temporal progression in automated quantification in echocardiography. Adapted from Nolan et al. (10). Permission obtained from the publisher.

it has not occurred due to the cumbersome and time-consuming nature of the approach (35). The integration of ML algorithms can streamline the clinical workflow by automating numerous calculations (9).

Samad et al. utilized clinical and echocardiographic variables using a random forest model which achieved superior prediction accuracy (all AUC >0.82) over common clinical risk scores $(\mathrm{AUC}=0.69-0.79)$ and logistic regression models $(p<0.001)$ in 171,510 patients for predicting all-cause mortality (36). Zhang et al. trained a (CNN) which successfully identified views $(96 \%$ for parasternal long axis or $84 \%$ accuracy overall) with image segmentation accuracy reaching 72-90\% (37). Sanchez-Martinez et al. assessed velocity patterns to differentiate heart failure preserved ejection fraction (HFpEF) from healthy patients with encouraging results $(\kappa, 72.6 \%$; 95\% confidence interval, 58.187.0) (38). Similarly, Tabassian et al., classified phenotypes of HFpEF patients with symptomatology using strain parameters (asymptomatic vs. symptomatic; AUC $=0.89$; accuracy = $85 \%$; sensitivity $=86 \%$, specificity $=82 \%$ ) (39). Unsupervised clustering models in HFpEF patients have successfully predicted hospitalization risk, exercise intolerance, and LV filling pressure (40-43). Lancaster et al. applied clustering ML model which isolated diastolic dysfunction in 559 of 866 patients with 2 distinct groups, revealed moderate agreement with conventional classification (kappa $=0.41, p<0.001$ ) (44). Asch examined ML automated echocardiographic quantification of left ventricular ejection fraction (LVEF), there was an excellent agreement with reference values $(r=0.95)$ (45). Benjamin et al. applied deep learning which showed lung Doppler signals (LDS) predicted echocardiographic E/e' measurements $[r=0.67$ (admission) and 0.83 (discharge), $p<0.0001$ ] in 99 acute HF patients and lower event-free survival in high predicted- E/e' group $\mathrm{HF}$ patients with reduced EF $(P=0.0247)$ (46). Kusunose et al. showed deep learning was better than residents in detecting wall motion abnormalities (WMA) (AUC 0.99 vs. $0.90, p=0.002$ ) and WMA territories (AUC $=0.97$ vs. $0.83, p=0.003$ ), the ML architecture had a similar performance to experienced cardiologists (47). Similarly, Kusononse used deep learning to demonstrate similar ML derived LVEF with validation group $(r=0.82 \pm 0.02, p$ $<0.001$ ) (48). Donal utilized a random forest model to assess response to cardiac resynchronization therapy, best performance was obtained with strain measurements (AUC of 0.686) and QRS duration (AUC of 0.668) (49).

Topological data analysis (TDA) is a form of unsupervised learning which uses clustering to create network and shape (50). Casaclang-Verzosa et al. employed TDA to discern precise LV phenotypic recognition in severe aortic stenosis (AS) patients, it formed a loop (Figure 2) of mild and severe aortic stenosis on the right and left side $(p<0.001)$ which was linked by moderate AS on top and bottom sides with reduced and preserved ejection fraction $(p<0.0001)$ (51). Other centers have used TDA in AS patients similarly, heart failure patients, and for 

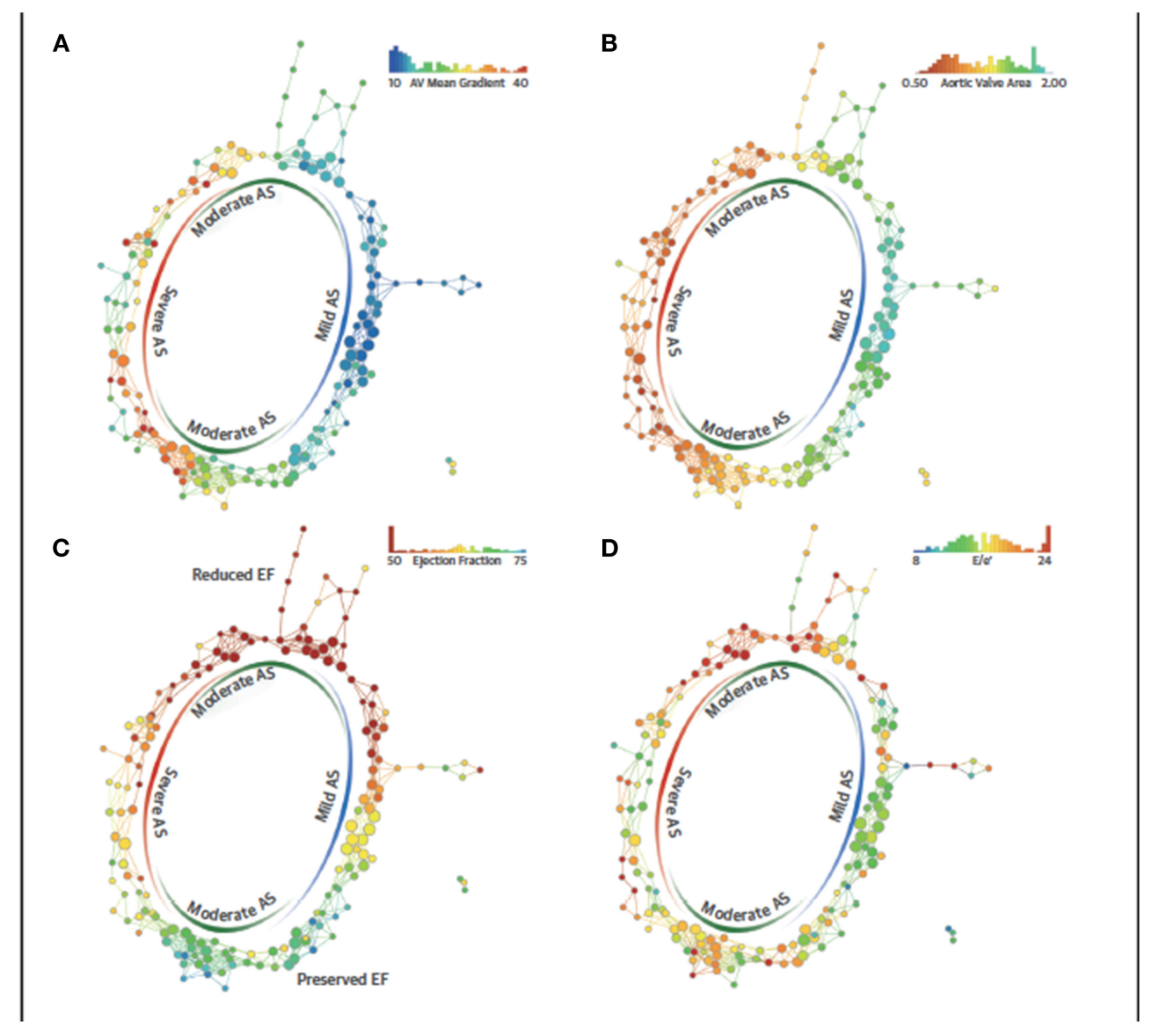

FIGURE 2 | Topological data analysis loop in aortic stenosis. The similarity network draws aggregation of patients with severe and mild AS in 2 opposite ends and moderate AS in the top and bottom arms of the loop. The red color represents the abnormal values, whereas blue represents the normal or minor malady in the disease space. (A) Spectrum of mean gradient in humans identified in the network of disease space. High gradients aggregated on the left side, whereas the low gradients were on the right side with moderate gradients in the middle. (B) Distribution of valve area on the network, (C) patient similarity network revealing preserved and reduced ejection fraction (EFs) in the patients with aortic stenosis (AS). The top arm clustered patients with the reduced EF, whereas the bottom arm described the patients with preserved EF. The red nodes consists of patients with EFs of $\leq 50 \%$, whereas the blue nodes consists of patients with EFs of $\geq 65 \%$. (D) Distribution of E/e' as a feature of diastolic dysfunction revealing the pattern of E/e' around moderate and severe AS. TDA, topological data analysis. Adapted from

Casaclang-Verzosa et al. (51). Permission obtained from the publisher.

predicting major adverse cardiovascular events (MACE) in large cohorts (52-54).

Several cardiovascular diseases can affect the cardiac valve. Previously surgical techniques were the gold standard techniques in valve intervention. With the advent of transcatheter approaches, there has been a paradigm shift in these approaches. The application of ML algorithms can help in the gradient and assessment of valvular heart disease. Also, they can help in therapeutic planning. Currently, is it still in the early stages. Costa et al. utilized deep learning to segment mitral valves in PLAX and apical 4 chamber views (55). Grady examined the role of ML for automating the proximal isovelocity surface area (PISA) assessment on echocardiography, it had excellent correlation with findings on magnetic resonance imaging (56). Wang et al. applied ML for evaluating mitral inflow and aortic outflow (57). Abdul Ghaffar et al. evaluated the role of semisupervised learning for phenogrouping based risk assessment in transcatheter aortic valve replacement (TAVR) (58). Group 5 was associated with significant in-hospital cardiovascular mortality (OR 3.5, $p=0.001)$.

\section{ROLE OF AI IN COMPUTED TOMOGRAPHY}

In recent years, computed tomography (CT) has emerged as a prominent technique in the field of cardiovascular imaging $(6,59,60)$, in part due to negative predictive value $(61)$. The evolution of scanner technology has led to drastic improvements in spatial and temporal resolution $(60,62)$. ML algorithms can automate and expedite many processes which will expand the frontiers of cardiac CT (5).

$\mathrm{CT}$ fractional flow reserve (CT- FFR) is arising as a noninvasive alternative in diagnosing chest pain. Though in the early stages of clinical implementation, it is one of the few methods to provide an anatomical and functional assessment. ML algorithms can compute FFR without computational fluid 
dynamics and provide additional prognostic information (6). Zhou et al. investigated the role of CT fractional flow reserve (CT FFR) for predicting myocardial bridge formation by multiple ML algorithms, ML selected features had higher AUC (0.75 \pm 0.04$)$ than clinical features $(0.53 \pm 0.09, p<0.0001)$, morphological features $(0.59 \pm 0.06, p=0.0025)$, and CT- FFR features $(0.62$ $\pm 0.06, p=0.0127$ ) (63). Coenen et al. compared CT- FFR with computational fluid dynamics and ML-derived CT- FFR for detecting coronary artery disease (CAD) through deep learning, there was an excellent correlation between both techniques $(R=$ 0.997) (64). Tang et al. noted that a novel on-site computational fluid dynamics-based CT FFR was better than CTA and invasive angiography in detecting lesion-specific ischemia, especially in intermediate lesions ( $p<0.001$ for all) [(65); Figure 3].

The presence of extensive coronary calcium (CAC) is the predominant issue in CAD evaluation (62). Furthermore, CAC can lead to overestimation of coronary vessel stenosis $(66,67)$. Al'Aref et al. utilized an ML model integrating clinical factors in conjunction with calcium score in the CONFIRM registry for predicting coronary artery disease in 35, 281 patients with CTA (68). They demonstrated superior AUC for ML and (CAC) (0.881) to ML alone (0.773), coronary calcium (0.886), and updated Diamond- Forrester Score (0.682). Similarly, Tesche et al. showed ML CT fractional flow reserve (FFR) was better than CTA alone in CAC, there was a significant difference in performance as Agatston scores increased (High scores- $p=0.04$, low to intermediate scores- $p<0.001$ ) (69). Kay et al. applied $\mathrm{ML}$ algorithms and radiomics to detect phenotypic information about high risk left ventricular hypertrophy (LVH) in CT with coronary artery calcium (CAC) scoring, these algorithmic models were highly effective in LVH detection (70). Hou et al. utilized supervised learning to calculate pretest probability in 6274 patients from CTA, the ML model had significantly higher discrimination for obstructive CAD than modified DiamondForester score and CAD consortium score ( 0.801 vs. 673 vs. 669 , $p<0.001)(71)$.

ML architectures have been used in a variety of different situations in CT. Baskaran et al. applied deep learning which verified with manual annotation for left ventricular volume $(r=0.98)$, right ventricular volume $(r=0.97)$, and atrial volumes in CT angiography $(\mathrm{CTA})(P<0.05)$ in 166 patients (72). Oikonomou et al. utilized a random forest model to predict cardiac risk from the radiomic profile of coronary perivascular adipose tissue (PVAT) in CTA, the fat radiomic profile (FRP) were able to augment MACE prediction beyond conventional risk stratification scores (C statistic $-0.126, p<$ 0.001) (73). Beecy et al. explored the role in acute ischemic stroke identification on CT, the AUC for the ML model was 0.91 for automated diagnosis of infarction and had a 93\% diagnostic accuracy with expert physician interpretation (74). Al'Aref et al. investigated the potential of supervised learning in CTA to identify culprit lesion precursors from acute coronary syndrome patients, the ML model displayed higher AUC for distinguishing precursors than multiple other models (0.774 vs. 0.599 vs. 0.532 vs. $0.672, p<0.01$ ) (75). Eisenberg used deep learning to show epicardial adipose tissue $(\mathrm{EAT})$ volume $(\mathrm{HR} 1.35, p<0.01)$ predicted MACE on CT, while attenuation $(0.83, p=0.01)$ had an inverse relationship (76).

\section{ROLE OF AI IN NUCLEAR CARDIOLOGY}

Single-photon emission computed tomography (SPECT) myocardial perfusion imaging (MPI) is the cardinal test in nuclear cardiology, plays a paramount role in the assessment of obstructive CAD (17). SPECT is predominantly used to evaluate myocardial perfusion and to identify possible perfusion defects either during rest or stress imaging indicating underlying ischemia (17). There are significant disparities in the diagnostic performance of SPECT attributed to many aspects that can be addressed by ML (77).

Betancur demonstrated deep learning was superior to total perfusion deficit (TPD) in MPI for CAD prediction $(78,79)$. With unsupervised learning, Betancur et al. exhibited higher (MACE) compared to expert readers, automated total perfusion deficit (TPD), and automated ischemic perfusion deficit in SPECT MPI and clinical factors for 2619 patients (AUC: 0.81 vs. 0.65 vs. 0.73 vs. $0.71, p<0.01$ for all) (80). Hu et al. explored the role of ML networks in automatic rest scan cancellation and prognostic safety, patients selected for rest scan cancellation had lower annualized MACE rates than the physician or clinical selection rules (all, $P<0.0001$ ) (81). Otaki et al. compared ML with visual reading for predicting MACE in 19,495 patients, it enabled more precise risk stratification than visual analysis (82). Juarez-Orozco et al. assessed the role of deep learning in 1,185 patients for polar maps in ischemia by positron emission tomography (PET), deep learning had an AUC of $0.90 \pm 0.02$ and outperformed all comparator models (all pairwise $p<0.01$ ) (83). $\mathrm{Hu}$ et al. investigated the ML algorithm to per- vessel prediction of early coronary revascularization within 90 days of SPECT MPI, they found ML AUC was superior to regional stress TPD, combined- view TPD, and ischemic TPD (0.79 vs. 0.71 vs. 0.72 , $P<0.001)(84)$.

\section{ROLE OF AI IN CARDIAC MAGNETIC RESONANCE IMAGING}

Over the last several years, cardiac magnetic resonance imaging (CMR) has emerged as an indispensable tool in the field of cardiovascular imaging (12). Substantial strides in the technological front have enhanced the capability of CMR for risk stratification and diagnosis. CMR is heralded as the gold standard for non-invasive assessment of the ejection fraction and left ventricular volume (27). Furthermore, it enables tissue characterization which can dictate medical management (85). Similar to echocardiography, strain is an emerging biomarker that can help in the ascension of $\operatorname{CMR}(86,87)$. Nevertheless, some processes in CMR take substantial time such as measuring volume or contour tracing (86). The integration of ML architectures can help expand the domain of CMR and transcend into new frontiers in cardiovascular imaging.

Ruijsink et al. presented exceedingly high CNN algorithm correlation with a manual analysis of LV and right ventricular 


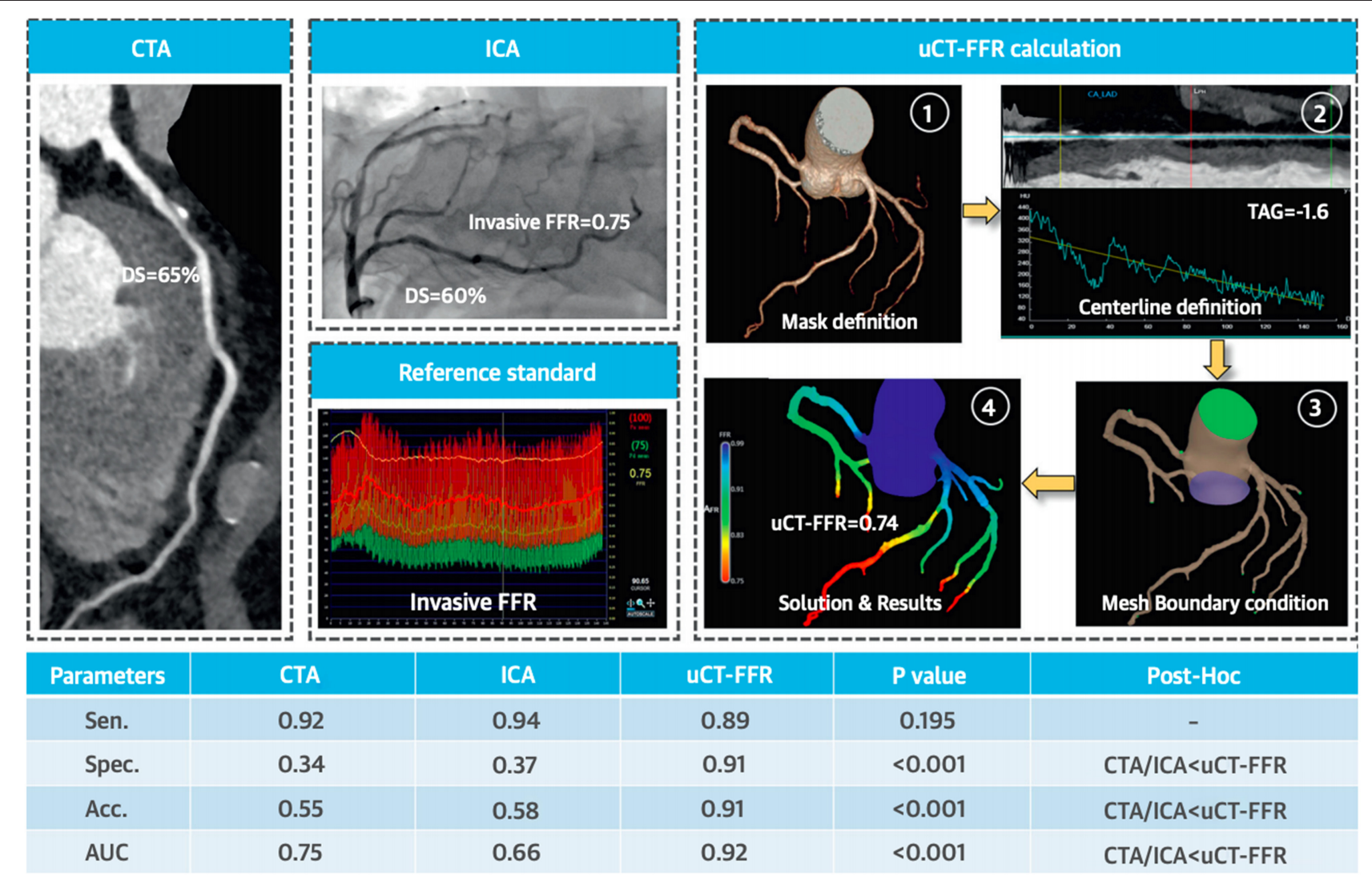

FIGURE 3 | Functional significance of proximal left anterior descending artery stenosis. Adapted from Tang et al. (65). Permission obtained from the publisher.

volumes (all $r>0.95$ ), strain (circumferential $r=0.89$, longitudinal $r>0.89$ ), and filling and ejection rate (all $r \geq 0.93$ ) in CMR (88). Winter et al. showed deep learning achieved a similar or higher performance with human experts for automatic segmentation of the right and left ventricular endocardium and epicardium (89). Bhuva et al. explored automated ML analysis of cardiac structures in CMR, automated analysis was 186 times faster than humans (0.07 vs. $13 \mathrm{~min})$ (90). Jain et al. utilized ML to demonstrate phasic right atrial (RA) phasic is predictive of all-cause death in patients with and without $\mathrm{HF}$, adjusted RA reservoir strain ( $\mathrm{HR}=0.66, p=0.0154)$, $\mathrm{RA}$ conduit strain $(\mathrm{HR}=0.58, p=0.0039)$, and $\mathrm{RA}$ conduit strain rate $(\mathrm{HR}=1.51, p=0.0373)$ independently predicted all-cause mortality (91). Fahmy et al. investigated the role of deep learning in CMR scar quantification for hypertrophic cardiomyopathy, there was strong correlation between automatic and manual segmented scar volumes $(r=0.9, p<0.001$ per patient and $(r=0.84, p<0.001$ per slice $)$ and LV mass $(r=0.96, p<0.001$ per-patient and $r=0.93, p<$ 0.001) (92). Neisius applied ML and radiomics to differentiate hypertensive heart disease and hypertrophic cardiomyopathy with native $\mathrm{T} 1$ mapping. The selected texture attributes in conjunction with the support vector machine classifier provided maximal diagnostic accuracy (c statistic -0.820) in comparison to T1 mapping (c statistic - 0.549) for distinguishing between the two entities (93). Knott et al. studied the role of AIbased quantification of stress myocardial blood flow (MBF) and myocardial perfusion reserve (MPR) in CMR for CAD, reduced quantities of both factors were independently associated with death and MACE (94). Swift et al. utilized ML in CMR to extract features and automated pulmonary artery hypertension (PAH) diagnosis, the AUC of the diagnostic approach was superior to CMR metrics (0.92 for PAH, $p<0.001$ ) and less time consuming (95).

\section{DISCUSSION}

\section{Our Contemporary Views on Artificial Intelligence and Machine Learning}

$\mathrm{CAD}$ is one of the leading causes of mortality in the world and is responsible for a host of cardiovascular-related complications (59, 96, 97). Although it may be relatively easy to pinpoint the exact cause of death but implementing universal solutions in terms of medications or intervention is not necessarily straightforward. There is a fundamental concept present at hand, it must be greatly emphasized that cardiovascular disease is heterogeneous in nature (98). The pathophysiology of cardiovascular disease encompasses various interactions between 
etiological factors, molecular components, genetic attributes, and intricate pathways (98). This is further compounded by varying clinical presentations which further complicate diagnosis and prognostication (22). These clinical dilemmas underpin the necessity and integration of ML frameworks in imaging or clinical pipelines in cardiovascular care (13). ML algorithms can extrapolate information from these multi-dimensional matrices to delineate unique patterns not witnessed before (24). Cloudbased infrastructures enhance data collection allowing for individualized care (20). Many studies have already shown the superiority of big data ML algorithms over standard care in areas including heart failure, AS, and ischemic heart disease (Table 2). By harvesting this information routinely, we can customize individualized solutions for medical management. AI can truly usher the era of precision medicine into modern-day medicine $(1,99)$.

The integration of genomics into $\mathrm{ML}$ algorithms will be more beneficial than popular risk scores which are restricted to a few variables. The Framingham Risk Score, for instance, is widely utilized for this purpose but does not have a genetic aspect. Genomics is being increasingly integrated into clinical databases or pan-omic datasets (98). Furthermore, they can provide valuable insight into underlying pathophysiology in various cardiac diseases (23). Among all current approaches, ML algorithms can truly appreciate the depth of information present dormant in these datasets $(13,30)$. This can lead to the development of new biomarkers or potential drug pathways $(22,23)$. In the coming days, combing cardiovascular imaging with pan-omic techniques will become the eventual standard in patient care (23). Also, this new information can help better stratify patient populations appropriately (8).

The rise of radiomics will be catapulted by the rise of ML architectures (20). Radiomics allows to extract of more features from imaging in greater detail and facilitates quantitative assessment. These features can be measured and evaluated. They can be particularly advantageous in various heterogeneous conditions. It can also help distinguish between various pathological entities that appear similar to imaging. Furthermore, it can be used to detect certain phenotypes within these conditions (100).

AI is driving the current philosophy in research to evolve and be flexible. The current norm of research is very linear, moving from a hypothesis to a conclusion (14). In reality, our lives and even our pathophysiology are actually multilinear. ML algorithms can analyze data in a variety of manners, we should be able to modify our hypothesis accordingly (23). This allows our research to be very dynamic and this can lead to new data-driven discoveries (14). This mindset may be needed as we move forward with the integration of $\mathrm{ML}$ algorithms in cardiovascular imaging.

AI can even improve randomized clinical trials (RCTS) in clinical research and cardiovascular imaging. The findings from RCTS define clinical care and are incorporated into national or international guidelines. Many pivotal RCTs fail to reach enrollment goals or have lofty expectations (101). If AI can analyze preliminary results from clinical trials, investigators can use this information appropriately (102). Better classification of the disease in question will allow the superior design of the RCT, there will be a more precise definition of the underlying condition. ML algorithms can determine which patient profiles may predict response to treatment or susceptibility to complications, this will enable better enrollment strategies (102). The early analysis can be used to redesign the trial or not conduct the trial in the first place which can save resources and time. Implementation of ML algorithms can also augment randomization by introducing additional imaging or clinical attributes (101).

The benefits of AI and ML algorithms will trickle downwards toward peripheral or community level hospitals. Besides, it can have significant advantages in the imaging lab setting also. AI can automatically develop complex protocols in cardiovascular imaging (Figure 4). Furthermore, it has the capability of adjusting these protocols depending on the situation. It can reduce errors in the acquisition, automate measurements, and greatly improve efficiency (11). This will result in increased decision support and confidence in imaging findings (11). As a result, this can help standardize reporting and improve the overall process. Recent developments are showing the promise of $\mathrm{AI}$ in prediction during intervention and management. AI can be used in combination with virtual planning to create digital twins (103). Interventional treatment can be simulated on twin which can plan actual intervention. This can lead to a number of individualized treatment options.

\section{Potential Issues in Machine Learning}

Though the potential of ML algorithms is tremendous, several looming issues need to be addressed for successful integration (12). For any ML algorithm to thrive, it needs adequate exposure to large data sets. This is simpler said than done. Many hurdles need to be overcome before procuring these unique data sets. Institutions must learn to share data among themselves or have some form of agreement in place. Furthermore, multiple institutional review boards (IRBs) are required to share data and it can be a tedious process (17). Besides, data sets need to be deidentified to maintain patient security. If these datasets can be made publicly accessible, then centers can benefit.

Multiple imaging storing systems exist within institutions which include picture archive and communication systems (PACS) or digital imaging and communications in medicine (DICOM). Each academic center may have different protocols in place. A universal data standard needs to be recognized and implemented with minimal variation (104). This will enable the growth of ML in various institutions (104).

The potential for false discovery is a potential pitfall with ML algorithms $(12,25)$. This can occur with smaller data sets. Investigators need to clearly define the purpose of their research before they interact with ML algorithms. Besides, Investigators need to be constantly vigilant to prevent unintentional biases from creeping into the model. Sampling bias can occur if the data does not capture the heterogeneity of cardiovascular disease. Unintentional prejudicial biases can be introduced into the model. One also must be aware of measurement bias as well. Frequent discussions are needed between the 
TABLE 2 | Recent examples of studies applying machine learning in cardiovascular interpretation.

\begin{tabular}{|c|c|c|c|}
\hline Study & ML approach & Imaging type & Brief Study description \\
\hline Samad et al. (36) & Supervised learning & Echo & To predict survival by using clinical and echocardiographic data \\
\hline Zhang et al. (37) & Deep learning & Echo & To achieve automatic interpretation with echocardiographic data \\
\hline Sanchez-Martinez et al. (38) & Unsupervised learning & Echo & To examine differences between HFpEF and healthy patients \\
\hline Tabassian et al. (39) & Supervised learning & Echo & $\begin{array}{l}\text { To identify patients with HFpEF through spatiotemporal variations of strain during } \\
\text { stress and exercise }\end{array}$ \\
\hline Mishra et al. (40) & Unsupervised learning & Echo & To identify clusters of HF patients and the risk of HF hospitalization \\
\hline Przewlocka-Kosmala et al. (41) & Unsupervised learning & Echo & To identify clusters of HFpEF patients \\
\hline Omar et al. (42) & Unsupervised learning & Echo & $\begin{array}{l}\text { To perform cluster analysis of left atrial and left ventricular strain in diastolic } \\
\text { dysfunction patients }\end{array}$ \\
\hline Salem Omar et al. (43) & Supervised learning & Echo & To characterize left ventricular filling pressure in diastolic dysfunction patients \\
\hline Lancaster et al. (44) & Unsupervised learning & Echo & To cluster echocardiographic variables to isolate high-risk phenotyping patterns \\
\hline Asch et al. (45) & ML algorithm & Echo & To examine automatic quantification of ejection fraction \\
\hline Benjamin et al. (46) & Deep learning & Echo & $\begin{array}{l}\text { To examine the relationship between lung Doppler signal with mitral E'/e ratio and } \\
\text { outcomes }\end{array}$ \\
\hline Kusunose et al. (47) & Deep learning & Echo & To detect wall motion abnormalities \\
\hline Kusunose et al. (48) & Deep learning & Echo & To automate LVEF \\
\hline Donal et al. (49) & Supervised learning & Echo & To assess response to cardiac resynchronization therapy \\
\hline Casaclang-Verzosa et al. (51) & Unsupervised learning & Echo & To identify unique phenotypes during AS progression \\
\hline Kwak et al. (53) & Unsupervised learning & Echo & To identify which AS clusters are associated with cardiovascular complications \\
\hline Tokodi et al. (54) & Unsupervised learning & Echo & To detect clusters of patients and predict MACE events \\
\hline Cho et al. (52) & Unsupervised learning & Echo & To identify clusters of heart failure patients and predict cardiovascular complications \\
\hline Baskaran et al. (72) & Deep learning & CT & $\begin{array}{l}\text { To compare the automatic and manual assessment of left and right heart sided } \\
\text { structures and function }\end{array}$ \\
\hline Zhou et al. (63) & Multiple ML algorithms & CT & To utilize CT FFR to predict myocardial bridge formation \\
\hline Oikonomou et al. (73) & Supervised learning & CT & To assess the potential of perivascular fat in cardiac risk prediction \\
\hline Beecy et al. (74) & Deep learning & CT & To identify acute ischemic stroke in CT \\
\hline Al'Aref et al. (75) & Supervised learning & CT & To detect culprit coronary lesions in CT for acute coronary syndrome patients \\
\hline Coenen et al. (64) & Supervised learning & CT & To detect CAD \\
\hline Kay et al. (70) & ML algorithm & CT & To detect phenotypic information about left ventricular hypertrophy \\
\hline Eisenberg et al. (76) & Deep learning & CT & To assess the role of epicardial tissue in MACE events \\
\hline Al'Aref et al. (68) & Multiple ML algorithm & CT & To use coronary calcium and clinical factors to predict CAD \\
\hline Tesche et al. (69) & ML algorithm & CT & To compare ML CT FFR and CT and CAC \\
\hline Tang et al. (65) & ML algorithm & CT & To compare ML CT FFR with CTA and invasive angiography \\
\hline Hou et al. (71) & Supervised learning & CT & To calculate the pre-test probability of CAD \\
\hline Betancur et al. (78) & Deep learning & Nuclear & To assess CAD prediction \\
\hline Betancur et al. (79) & Deep learning & Nuclear & To assess CAD prediction in semi-upright and supine stress MPI \\
\hline Betancur et al. (80) & Supervised learning & Nuclear & To compare MACE predictive accuracy Of ML with expert evaluation \\
\hline Hu et al. (81) & ML algorithm & Nuclear & $\begin{array}{l}\text { To compare rest scan cancellation rates and complications between ML algorithm } \\
\text { and physician or clinical systems }\end{array}$ \\
\hline Otaki et al. (82) & ML algorithm & Nuclear & To compare MACE predictive accuracy of visual reading with ML networks \\
\hline Juarez-Orozco et al. (83) & Deep learning & Nuclear & To assess the role of deep learning in polar maps for ischemia \\
\hline Hu et al. (84) & ML algorithm & CMR & To predict per-vessel prediction of early coronary revascularization in SPECT MPI \\
\hline Ruijsink et al. (88) & Deep learning & CMR & To compare automatic ventricular measurements with CMR manually \\
\hline Winther et al. (89) & Deep learning & CMR & To evaluate automatic segmentation of epicardium and endocardium by deep learning \\
\hline Bhuva et al. (90) & Deep learning & CMR & To evaluate automated analysis \\
\hline Jain et al. (91) & ML algorithm & CMR & To evaluate right atrial phasic function in predicting all-cause death \\
\hline Fahmy et al. (92) & Deep learning & CMR & To estimate CMR scar quantification in hypertrophic cardiomyopathy \\
\hline Neisius et al. (93) & Supervised learning & CMR & To differentiate hypertrophic cardiomyopathy and hypertensive heart disease \\
\hline Knott et al. (94) & Al algorithm & CMR & Al-based quantification of myocardial blood flow and myocardial perfusion reserve \\
\hline Swift et al. (95) & Multiple ML algorithm & CMR & To extract features and automate PAH diagnosis \\
\hline
\end{tabular}

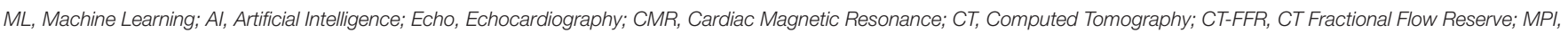

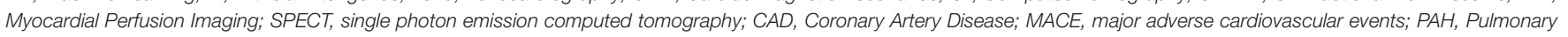
Artery Hypertension; CAC, Coronary Artery Calcium. 


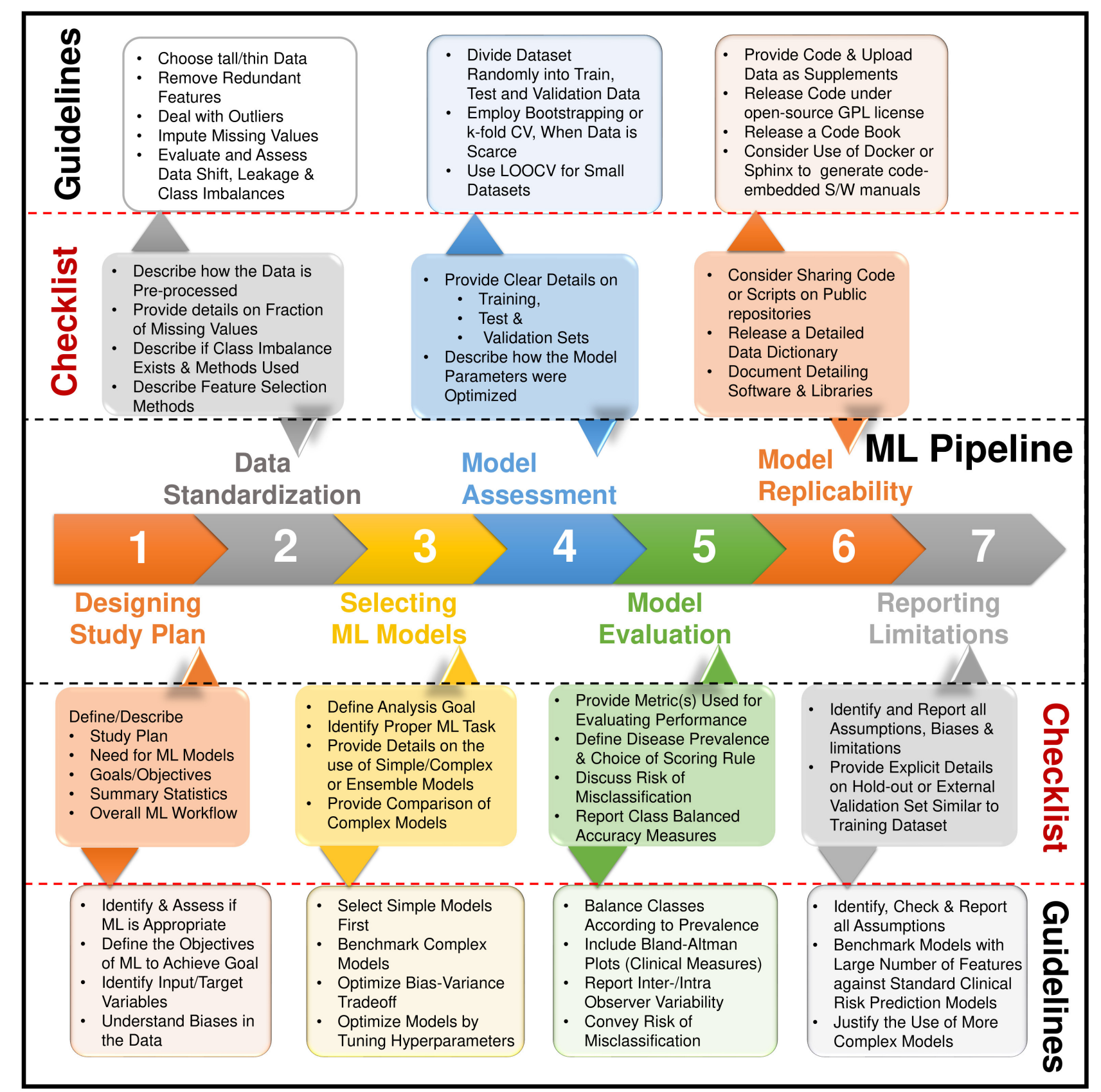

FIGURE 4 | Steps for building a machine learning pipeline and the reporting items in a checklist. Adapted from Sengupta et al. (2).

physician and engineers before ML initiation in various research endeavors (18).

The "black box" nature has always been the Achilles heel of ML algorithms and has been a deterrent in its adoption. These algorithms are not programmed to have ethics. For ML algorithms to truly advance in the medical field, physicians need to be properly educated about these topics. Medical school curriculums should introduce ML to medical students, so they have adequate exposure (67). Once they complete their medical training, they can be well-versed and conduct proper research (67).

\section{FUTURE DIRECTIONS OF MACHINE LEARNING}

Telemedicine has experienced phenomenal growth in recent years due to miniaturized equipment and wearable devices (3, 
105). With the evolution of smartphone applications, this will have a revolutionary impact on medical management (4). With infrastructures, these devices and applications can deliver clinical care to underserved regions throughout the world. We have had positive experiences with handheld ultrasound with cloud technology integration in remote regions of India $(106,107)$. The data arising from these devices cannot be adequately analyzed by current statistical approaches, it will only be possible with ML algorithms (3).

In parallel with the growth of telemedicine and mhealth, the rapid advances in technology can have a fundamental impact on various healthcare business models. This will lead to the eventual development of "smart" clinics. These clinics usually have an array of miniaturized devices such as pocket ultrasound and smartphone applications. These services will be linked to $\mathrm{AI}$ or $\mathrm{ML}$ algorithm-driven operations that can analyze information in real-time. This will allow precision medicine to be delivered in each admission or routine

\section{REFERENCES}

1. Johnson KW, Torres Soto J, Glicksberg BS, Shameer K, Miotto R, Ali M, et al. Artificial intelligence in cardiology. J Am Coll Cardiol. (2018) 71:2668-79. doi: 10.1016/j.jacc.2018.03.521

2. Sengupta PP, Shrestha S, Berthon B, Messas E, Donal E, Tison GH, et al. Proposed requirements for cardiovascular imaging-related machine learning evaluation (PRIME): a checklist: reviewed by the American college of cardiology healthcare innovation council. JACC Cardiovasc Imaging. (2020) 13:2017-35. doi: 10.1016/j.jcmg.2020.07.015

3. Seetharam K, Kagiyama N, Sengupta PP. Application of mobile health, telemedicine and artificial intelligence to echocardiography. Echo Res Pract. (2019) 6:R41-52. doi: 10.1530/ERP-18-0081

4. Magnani JW, Mujahid MS, Aronow HD, Cene CW, Dickson VV, Havranek E, et al. Health literacy and cardiovascular disease: fundamental relevance to primary and secondary prevention: a scientific statement from the American heart association. Circulation. (2018) 138:e4874. doi: 10.1161/CIR.0000000000000579

5. Dey D, Slomka PJ, Leeson P, Comaniciu D, Shrestha S, Sengupta PP, et al. Artificial intelligence in cardiovascular imaging: JACC state-of-theart review. J Am Coll Cardiol. (2019) 73:1317-35. doi: 10.1016/j.jacc.2018. 12.054

6. Al'Aref SJ, Anchouche K, Singh G, Slomka PJ, Kolli KK, Kumar A, et al. Clinical applications of machine learning in cardiovascular disease and its relevance to cardiac imaging. Eur Heart J. (2018) 40:197586. doi: 10.1093/eurheartj/ehy404

7. Shrestha S, Sengupta PP. Imaging heart failure with artificial intelligence: improving the realism of synthetic wisdom. Circ Cardiovasc Imaging. (2018) 11:e007723. doi: 10.1161/CIRCIMAGING.118.007723

8. Collet C, Penicka M. Integrating patient-specific information in the noninvasive quest for the vulnerable patient. JACC Cardiovasc Imaging. (2019) 12:1998-9. doi: 10.1016/j.jcmg.2018.10.025

9. Shrestha S, Sengupta PP. The mechanics of machine learning: from a concept to value. J Am Soc Echocardiogr. (2018) 31:1285-7. doi: 10.1016/j.echo.2018.10.003

10. Nolan MT, Thavendiranathan P. Automated quantification in echocardiography. JACC Cardiovasc Imaging. (2019) 12:107392. doi: 10.1016/j.jcmg.2018.11.038

11. Sengupta PP, Adjeroh DA. Will artificial intelligence replace the human echocardiographer? Circulation. (2018) 138:163942. doi: 10.1161/CIRCULATIONAHA.118.037095 follow up. These clinics will be integral to the field of cardiovascular imaging.

\section{CONCLUSION}

The profound impact of AI in cardiovascular imaging will have monumental effects on clinical care. ML algorithms will connect information from multiple sources in a seamless transition. It will automate several tasks which will provide more time for patient interactions for cardiologists. It will greatly augment the workflow and ultimately improve medical management. AI and ML-driven algorithms are no longer a possibility but an inevitability in the field of cardiovascular imaging.

\section{AUTHOR CONTRIBUTIONS}

All authors listed have made a substantial, direct and intellectual contribution to the work, and approved it for publication.

12. Seetharam K, Shrestha S, Sengupta PP. Artificial intelligence in cardiovascular medicine. Curr Treat Options Cardiovasc Med. (2019) 21:25. doi: 10.1007/s11936-019-0728-1

13. Min JK. Chess and coronary artery ischemia. Circulation. (2018) 11:e007943. doi: 10.1161/CIRCIMAGING.118.007943

14. Sengupta PP, Shrestha S. Machine learning for data-driven discovery: the rise and relevance. JACC Cardiovasc Imaging. (2019) 12:6902. doi: 10.1016/j.jcmg.2018.06.030

15. Pennell D, Delgado V, Knuuti J, Maurovich-Horvat P, Bax JJ. The year in cardiology: imaging: the year in cardiology 2019. Eur Heart J. (2020) 41:739-47. doi: 10.1093/eurheartj/ehz930

16. Singh A, Falk RH. A new staging system for cardiac transthyretin amyloidosis: is it already on the verge of obsolescence? Eur Heart J. (2018) 39:2807-9. doi: 10.1093/eurheartj/ehx740

17. Seetharam K, Shresthra S, Mills JD, Sengupta PP. Artificial intelligence in nuclear cardiology: adding value to prognostication. Curr Cardiovasc Imaging Rep. (2019) 12:14. doi: 10.1007/s12410-0199490-8

18. van Assen M, Cornelissen LJ. Artificial intelligence: from scientific curiosity to clinical precocity? JACC Cardiovasc Imaging. (2020) 13:11724. doi: 10.1016/j.jcmg.2019.09.008

19. Omar AMS, Krittanawong C, Narula S, Narula J, Argulian E. Echocardiographic data in artificial intelligence research: primer on concepts of big data and latent states. JACC Cardiovasc Imaging. (2020) 13(1 Pt 1):170-2. doi: 10.1016/j.jcmg.2019.07.017

20. Al-Mallah MH. Radiomics in hypertrophic cardiomyopathy: the new tool. JACC Cardiovasc Imaging. (2019) 12:1955-7. doi: 10.1016/j.jcmg.2019.02.004

21. Seetharam K, Raina S, Sengupta P. The role of artificial intelligence in echocardiography. Curr Cardiol Rep. (2020) 22:99. doi: 10.1007/s11886-020-01329-7

22. Shameer K, Johnson KW, Glicksberg BS, Dudley JT, Sengupta PP. Machine learning in cardiovascular medicine: are we there yet? Heart. (2018) 104:1156-64. doi: 10.1136/heartjnl-2017-311198

23. Argulian E, Narula J. Imaging-verified disease stages: branching off into the landscape of possibilities. JACC Cardiovasc Imaging. (2020) 13:16713. doi: 10.1016/j.jcmg.2020.02.013

24. D'Hooge J, Fraser AG. Learning about machine learning to create a self-driving echocardiographic laboratory. Circulation. (2018) 138:16368. doi: 10.1161/CIRCULATIONAHA.118.037094

25. Seetharam K, Shrestha S, Sengupta P. Artificial intelligence in cardiac imaging. US Cardiol Rev. (2020) 13:110-6. doi: 10.15420/usc.2019.19.2 
26. Kagiyama N, Shrestha S, Farjo PD, Sengupta PP. Artificial intelligence: practical primer for clinical research in cardiovascular disease. J Am Heart Assoc. (2019) 8:e012788. doi: 10.1161/JAHA.119.012788

27. Seetharam K, Kagiyama N, Shrestha S, Sengupta P. Clinical inference from cardiovascular imaging: paradigm shift towards machine-based intelligent platform. Curr Treat Options Cardiovasc Med. (2020) 22:8. doi: 10.1007/s11936-020-0805-5

28. Bianco CM, Farjo PD, Ghaffar YA, Sengupta PP. Myocardial mechanics in patients with normal LVEF and diastolic dysfunction. JACC Cardiovasc Imaging. (2020) 13(1 Pt 2):258-71. doi: 10.1016/j.jcmg.2018.12.035

29. Seetharam K, Sengupta PP, Bianco CM. Cardiac mechanics in heart failure with preserved ejection fraction. Echocardiography. (2020) 37:193643. doi: 10.1111/echo.14764

30. Garratt KN, Schneider MA. Thinking machines and risk assessment: on the path to precision medicine. J Am Heart Assoc. (2019) 8:e011969. doi: 10.1161/JAHA.119.011969

31. Litjens G, Ciompi F, Wolterink JM, de Vos BD, Leiner T, Teuwen J, et al. State-of-the-art deep learning in cardiovascular image analysis. JACC Cardiovasc Imaging. (2019) $12(8 \mathrm{Pt}$ 1):1549-65. doi: 10.1016/j.jcmg.2019.06.009

32. Krittanawong C, Zhang H, Wang Z, Aydar M, Kitai T. Artificial intelligence in precision cardiovascular medicine. J Am Coll Cardiol. (2017) 69:265764. doi: 10.1016/j.jacc.2017.03.571

33. Krittanawong C, Johnson KW, Rosenson RS, Wang Z, Aydar M, Baber U, et al. Deep learning for cardiovascular medicine: a practical primer. Eur Heart J. (2019) 40:2058-73. doi: 10.1093/eurheartj/ehz056

34. Bizopoulos P, Koutsouris D. Deep learning in cardiology. IEEE Rev Biomed Eng. (2019) 12:168-93. doi: 10.1109/RBME.2018.2885714

35. Leeson P. (Deep) learning your left from your right. JACC Cardiovasc Imaging. (2020) 13(2 Pt 1):382-4. doi: 10.1016/j.jcmg.2019.03.015

36. Samad MD, Ulloa A, Wehner GJ, Jing L, Hartzel D, Good CW, et al. Predicting survival from large echocardiography and electronic health record datasets: optimization with machine learning. JACC Cardiovasc Imaging. (2019) 12:681-9. doi: 10.1016/j.jcmg.2018.04.026

37. Zhang J, Gajjala S, Agrawal P, Tison GH, Hallock LA, Beussink-Nelson L, et al. Fully automated echocardiogram interpretation in clinical practice. Circulation. (2018) 138:162335. doi: 10.1161/CIRCULATIONAHA.118.034338

38. Sanchez-Martinez S, Duchateau N, Erdei T, Kunszt G, Aakhus S, Degiovanni A, et al. Machine learning analysis of left ventricular function to characterize heart failure with preserved ejection fraction. Circ Cardiovasc Imaging. (2018) 11:e007138. doi: 10.1161/CIRCIMAGING.117.007138

39. Tabassian M, Sunderji I, Erdei T, Sanchez-Martinez S, Degiovanni A, Marino $\mathrm{P}$, et al. Diagnosis of heart failure with preserved ejection fraction: machine learning of spatiotemporal variations in left ventricular deformation. J Am Soc Echocardiogr. (2018) 31:1272-84.e9. doi: 10.1016/j.echo.2018.07.013

40. Mishra RK, Tison GH, Fang Q, Scherzer R, Whooley MA, Schiller NB. Association of machine learning-derived phenogroupings of echocardiographic variables with heart failure in stable coronary artery disease: the heart and soul study. J Am Soc Echocardiogr. (2020) 33:322-31.e1. doi: 10.1016/j.echo.2019.09.010

41. Przewlocka-Kosmala M, Marwick TH, Dabrowski A, Kosmala W. Contribution of cardiovascular reserve to prognostic categories of heart failure with preserved ejection fraction: a classification based on machine learning. J Am Soc Echocardiogr. (2019) 32:604-15.e6. doi: 10.1016/j.echo.2018.12.002

42. Omar AMS, Narula S, Abdel Rahman MA, Pedrizzetti G, Raslan H, Rifaie $\mathrm{O}$, et al. Precision phenotyping in heart failure and pattern clustering of ultrasound data for the assessment of diastolic dysfunction. JACC Cardiovasc Imaging. (2017) 10:1291-303. doi: 10.1016/j.jcmg.2016. 10.012

43. Salem Omar AM, Shameer K, Narula S, Abdel Rahman MA, Rifaie $\mathrm{O}$, Narula $\mathrm{J}$, et al. Artificial intelligence-based assessment of left ventricular filling pressures from 2-dimensional cardiac ultrasound images. JACC Cardiovasc Imaging. (2018) 11:509-10. doi: 10.1016/j.jcmg.201 7.05.003

44. Lancaster MC, Salem Omar AM, Narula S, Kulkarni H, Narula J, Sengupta PP. Phenotypic clustering of left ventricular diastolic function parameters: patterns and prognostic relevance. JACC Cardiovasc Imaging. (2018) $12(7 \mathrm{Pt}$ 1):1149-61. doi: 10.1016/j.jcmg.2018.02.005

45. Asch FM, Poilvert N, Abraham T, Jankowski M, Cleve J, Adams M, et al. Automated echocardiographic quantification of left ventricular ejection fraction without volume measurements using a machine learning algorithm mimicking a human expert. Circ Cardiovasc Imaging. (2019) 12:e009303. doi: 10.1161/CIRCIMAGING.119.009303

46. Benjamin MM, Bianco C, Caccamo M, Sokos G, Kagiyama N, Shrestha $\mathrm{S}$, et al. Non-invasive prediction of tissue Doppler-derived E/e' ratio using lung Doppler signals. Eur Heart J Cardiovasc Imaging. (2020) 21:9941004. doi: 10.1093/ehjci/jeaa090

47. Kusunose K, Abe T, Haga A, Fukuda D, Yamada H, Harada M, et al. A deep learning approach for assessment of regional wall motion abnormality from echocardiographic images. JACC Cardiovasc Imaging. (2020) $13(2 \mathrm{Pt}$ 1):374-81. doi: 10.1016/j.jcmg.2019.02.024

48. Kusunose K, Haga A, Yamaguchi N, Abe T, Fukuda D, Yamada H, et al. Deep learning for assessment of left ventricular ejection fraction from echocardiographic images. J Am Soc Echocardiogr. (2020) 33:6325.e1. doi: 10.1016/j.echo.2020.01.009

49. Donal E, Hubert A, Le Rolle V, Leclercq C, Martins R, Mabo P, et al. New multiparametric analysis of cardiac dyssynchrony: machine learning and prediction of response to CRT. JACC Cardiovasc Imaging. (2019) 12:18878. doi: 10.1016/j.jcmg.2019.03.009

50. Ng ACT, Delgado V, Bax JJ. Individualized patient risk stratification using machine learning and topological data analysis. JACC Cardiovasc Imaging. (2020) 13:1133-4. doi: 10.1016/j.jcmg.2020.02.003

51. Casaclang-Verzosa G, Shrestha S, Khalil MJ, Cho JS, Tokodi M, Balla S, et al. Network tomography for understanding phenotypic presentations in aortic stenosis. JACC Cardiovasc Imaging. (2019) 12:23648. doi: 10.1016/j.jcmg.2018.11.025

52. Cho J, Shrestha S, Kagiyama N, Hu L, Abdul Ghaffar Y, Casaclang-Verzosa $\mathrm{G}$, et al. A network-based 'phenomics' approach for discovering patient subtypes from high-throughput cardiac imaging data. JACC Cardiovasc Imaging. (2020) 13:1655-70. doi: 10.1016/j.jcmg.2020.02.008

53. Kwak S, Lee Y, Ko T, Yang S, Hwang IC, Park JB, et al. Unsupervised cluster analysis of patients with aortic stenosis reveals distinct population with different phenotypes and outcomes. Circ Cardiovasc Imaging. (2020) 13:e009707. doi: 10.1161/CIRCIMAGING.119.009707

54. Tokodi M, Shrestha S, Bianco C, Kagiyama N, Casaclang-Verzosa G, Narula J, et al. Interpatient similarities in cardiac function: a platform for personalized cardiovascular medicine. JACC Cardiovasc Imaging. (2020) 13:1119-32. doi: 10.1016/j.jcmg.2019.12.018

55. Costa E, Martins N, Sultan MS, Veiga D, Ferreira M, Mattos S, et al. Mitral valve leaflets segmentation in echocardiography using convolutional neural networks. In: 2019 IEEE 6th Portuguese Meeting on Bioengineering (ENBENG) Libson: IEEE. (2019). doi: 10.1109/ENBENG.2019.8692573

56. Grady L, Datta S, Kutter O, Duong C, Wein W, Little SH, et al. Regurgitation quantification using 3D PISA in volume echocardiography. In: International Conference on Medical Image Computing and Computer-Assisted Intervention. Quebec City, QC: Springer. (2011). doi: 10.1007/978-3-642-23626-6_63

57. Wang Y, Georgescu B, Datta S, Liu S, Vannan MA, Comaniciu D, et al. Automatic cardiac flow quantification on 3D volume color Doppler data. In: 2011 IEEE International Symposium on Biomedical Imaging: from Nano to Macro. Chicago, IL: IEEE. (2011). doi: 10.1109/ISBI.2011.5872729

58. Abdul Ghaffar Y, Osman M, Shrestha S, Shaukat F, Kagiyama N, Alkhouli $M$, et al. Usefulness of semi-supervised machine learningbased phenogrouping to improve risk assessment for patients undergoing transcatheter aortic valve implantation. Am J Cardiol. (2020) 136:12230. doi: 10.1016/j.amjcard.2020.08.048

59. Yeri A, Shah RV. Comparison of computational fluid dynamics and machine learning-based fractional flow reserve in coronary artery disease. Circulation. (2018) 11:e007950. doi: 10.1161/CIRCIMAGING.118. 007950

60. Nicol ED, Norgaard BL, Blanke P, Ahmadi A, Weir-McCall J, Horvat PM, et al. The future of cardiovascular computed tomography: advanced analytics and clinical insights. JACC Cardiovasc Imaging. (2019) 12:105872. doi: $10.1016 / j . j \mathrm{cmg} .2018 .11 .037$ 
61. Schoepf UJ, van Assen M. FFR-CT and CT myocardial perfusion imaging: friends or foes? JACC Cardiovasc Imaging. (2019) 12:24724. doi: 10.1016/j.jcmg.2019.03.011

62. Dey D, Lin A. Machine-learning CT-FFR and extensive coronary calcium: overcoming the Achilles heel of coronary computed tomography angiography. JACC Cardiovasc Imaging. (2020) 13:771-3. doi: 10.1016/j.jcmg.2019.08.011

63. Zhou F, Tang CX, Schoepf UJ, Tesche C, Rollins JD, Liu H, et al. Machine learning using CT-FFR predicts proximal atherosclerotic plaque formation associated with LAD myocardial bridging. JACC Cardiovasc Imaging. (2019) 12(8 Pt 1):1591-3. doi: 10.1016/j.jcmg.2019.01.018

64. Coenen A, Kim Y-H, Kruk M, Tesche C, Geer JD, Kurata A, et al. Diagnostic accuracy of a machine-learning approach to coronary computed tomographic angiography\&\#x2013; based fractional flow reserve. Circulation. (2018) 11:e007217. doi: 10.1161/CIRCIMAGING.117.007217

65. Tang CX, Liu CY, Lu MJ, Schoepf UJ, Tesche C, Bayer RR, et al. CT FFR for Ischemia-specific CAD with a new computational fluid dynamics algorithm: a Chinese Multicenter Study. JACC Cardiovasc Imaging. (2020) 13:980-90. doi: 10.1016/j.jcmg.2019.06.018

66. Budoff MJ, Dowe D, Jollis JG, Gitter M, Sutherland J, Halamert E, et al. Diagnostic performance of 64-multidetector row coronary computed tomographic angiography for evaluation of coronary artery stenosis in individuals without known coronary artery disease: results from the prospective multicenter ACCURACY (assessment by coronary computed tomographic angiography of individuals undergoing invasive coronary angiography) trial. J Am Coll Cardiol. (2008) 52:1724-32.

67. Sengupta PP, Shrestha S, Zeb I. Solving coronary risk: time to feed machines some calcium (score) supplements. Eur Heart J. (2020) 41:36870. doi: 10.1093/eurheartj/ehz708

68. Al'Aref SJ, Maliakal G, Singh G, van Rosendael AR, Ma X, Xu $\mathrm{Z}$, et al. Machine learning of clinical variables and coronary artery calcium scoring for the prediction of obstructive coronary artery disease on coronary computed tomography angiography: analysis from the CONFIRM registry. Eur Heart J. (2020) 41:359-67. doi: 10.1093/eurheartj/ ehz565

69. Tesche C, Otani K, De Cecco CN, Coenen A, de Geer J, Kruk M, et al. Influence of coronary calcium on diagnostic performance of machine learning CT-FFR: results from machine registry. JACC Cardiovasc Imaging. (2020) 13:760-70. doi: 10.1016/j.jcmg.2019.06.027

70. Kay FU, Abbara S, Joshi PH, Garg S, Khera A, Peshock RM. Identification of high-risk left ventricular hypertrophy on calcium scoring cardiac computed tomography scans: validation in the DHS. Circ Cardiovasc Imaging. (2020) 13:e009678. doi: 10.1161/CIRCIMAGING.119.009678

71. Hou ZH, Lu B, Li ZN, An YQ, Gao Y, Yin WH, et al. Machine learning for pretest probability of obstructive coronary stenosis in symptomatic patients. JACC Cardiovasc Imaging. (2019) 12:2584-6. doi: 10.1016/j.jcmg.2019. 07.030

72. Baskaran L, Maliakal G, Al'Aref SJ, Singh G, Xu Z, Michalak K, et al. Identification and quantification of cardiovascular structures from CCTA: an end-to-end, rapid, pixel-wise, deep-learning method. JACC Cardiovasc Imaging. (2019) 13:1163-71. doi: 10.1016/j.jcmg.2019. 08.025

73. Oikonomou EK, Williams MC, Kotanidis CP, Desai MY, Marwan M, Antonopoulos AS, et al. A novel machine learning-derived radiotranscriptomic signature of perivascular fat improves cardiac risk prediction using coronary CT angiography. Eur Heart J. (2019) 40:3529-43. doi: 10.1093/eurheartj/ehz592

74. Beecy AN, Chang Q, Anchouche K, Baskaran L, Elmore K, Kolli K, et al. A novel deep learning approach for automated diagnosis of acute ischemic infarction on computed tomography. JACC Cardiovasc Imaging. (2018) 11:1723-5. doi: 10.1016/j.jcmg.2018.03.012

75. Al'Aref SJ, Singh G, Choi JW, Xu Z, Maliakal G, van Rosendael AR, et al. A boosted ensemble algorithm for determination of plaque stability in highrisk patients on coronary CTA. JACC Cardiovasc Imaging. (2020)13:216373. doi: $10.1016 /$ j.jcmg.2020.03.025

76. Eisenberg E, McElhinney PA, Commandeur F, Chen X, Cadet S, Goeller $M$, et al. Deep learning-based quantification of epicardial adipose tissue volume and attenuation predicts major adverse cardiovascular events in asymptomatic subjects. Circ Cardiovasc Imaging. (2020) 13:e009829. doi: 10.1161/CIRCIMAGING.119.009829

77. Shrestha S, Sengupta PP. Machine learning for nuclear cardiology: the way forward. J Nucl Cardiol. (2019) 26:1755-8. doi: 10.1007/s12350-018-1284-x

78. Betancur J, Commandeur F, Motlagh M, Sharir T, Einstein AJ, Bokhari S, et al. Deep learning for prediction of obstructive disease from fast myocardial perfusion SPECT: a multicenter study. JACC Cardiovasc Imaging. (2018) 11:1654-63. doi: 10.1016/j.jcmg.2018.01.020

79. Betancur J, Hu LH, Commandeur F, Sharir T, Einstein AJ, Fish $\mathrm{MB}$, et al. Deep learning analysis of upright-supine high-efficiency SPECT myocardial perfusion imaging for prediction of obstructive coronary artery disease: a multicenter study. J Nucl Med. (2019) 60:66470. doi: 10.2967/jnumed.118.213538

80. Betancur J, Otaki Y, Motwani M, Fish MB, Lemley M, Dey D, et al. Prognostic value of combined clinical and myocardial perfusion imaging data using machine learning. JACC Cardiovasc Imaging. (2018) 11:10009. doi: 10.1016/j.jcmg.2017.07.024

81. $\mathrm{Hu} \mathrm{LH}$, Miller RJH, Sharir T, Commandeur F, Rios R, Einstein AJ, et al. Prognostically safe stress-only single-photon emission computed tomography myocardial perfusion imaging guided by machine learning: report from REFINE SPECT. Eur Heart J Cardiovasc Imaging. (2020) jeaa134. doi: 10.1093/ehjci/jeaa134. [Epub ahead of print].

82. Otaki Y, Betancur J, Sharir T, Hu LH, Gransar H, Liang JX, et al. 5-year prognostic value of quantitative versus visual MPI in subtle perfusion defects: results from REFINE SPECT. JACC Cardiovasc Imaging. (2020) 13:77485. doi: 10.1016/j.jcmg.2019.02.028

83. Juarez-Orozco LE, Martinez-Manzanera O, van der Zant FM, Knol RJJ, Knuuti J. Deep learning in quantitative PET myocardial perfusion imaging: a study on cardiovascular event prediction. JACC Cardiovasc Imaging. (2020) 13(1 Pt 1):180-2. doi: 10.1016/j.jcmg.2019.08.009

84. Hu LH, Betancur J, Sharir T, Einstein AJ, Bokhari S, Fish MB, et al. Machine learning predicts per-vessel early coronary revascularization after fast myocardial perfusion SPECT: results from multicentre REFINE SPECT registry. Eur Heart J Cardiovasc Imaging. (2020) 21:549-59. doi: 10.1093/ehjci/jez177

85. Penicka M. Mitral regurgitation flow variation: the end of a single time-point assessment? JACC Cardiovasc Imaging. (2019) 12:2443-4. doi: 10.1016/j.jcmg.2019.02.010

86. Judd RM. Machine learning in medical imaging: all journeys begin with a single step. JACC Cardiovasc Imaging. (2020) 13:696-8. doi: 10.1016/j.jcmg.2019.08.028

87. Hor KN, Gottliebson WM, Carson C, Wash E, Cnota J, Fleck R, et al. Comparison of magnetic resonance feature tracking for strain calculation with harmonic phase imaging analysis. JACC Cardiovasc Imaging. (2010) 3:144-51. doi: 10.1016/j.jcmg.2009.11.006

88. Ruijsink B, Puyol-Anton E, Oksuz I, Sinclair M, Bai W, Schnabel JA, et al. Fully automated, quality-controlled cardiac analysis from CMR: validation and large-scale application to characterize cardiac function. JACC Cardiovasc Imaging. (2019) 13:684-95. doi: 10.1016/j.jcmg.2019.05.030

89. Winther HB, Hundt C, Schmidt B, Czerner C, Bauersachs J, Wacker F, et al. v-net: deep learning for generalized biventricular mass and function parameters using multicenter cardiac MRI data. JACC Cardiovasc Imaging. (2018) 11:1036-8. doi: 10.1016/j.jcmg.2017.11.013

90. Bhuva AN, Bai W, Lau C, Davies RH, Ye Y, Bulluck H, et al. A multicenter, scan-rescan, human and machine learning CMR study to test generalizability and precision in imaging biomarker analysis. Circ Cardiovasc Imaging. (2019) 12:e009214. doi: 10.1161/CIRCIMAGING.119.009214

91. Jain S, Kuriakose D, Edelstein I, Ansari B, Oldland G, Gaddam S, et al. Right atrial phasic function in heart failure with preserved and reduced ejection fraction. JACC Cardiovasc Imaging. (2019) 12(8 Pt 1):146070. doi: 10.1016/j.jcmg.2018.08.020

92. Fahmy AS, Rausch J, Neisius U, Chan RH, Maron MS, Appelbaum E, et al. Automated cardiac MR scar quantification in hypertrophic cardiomyopathy using deep convolutional neural networks. JACC Cardiovasc Imaging. (2018) 11:1917-8. doi: 10.1016/j.jcmg.2018.04.030

93. Neisius U, Myerson L, Fahmy AS, Nakamori S, El-Rewaidy H, Joshi G, et al. Cardiovascular magnetic resonance feature tracking strain analysis for discrimination between hypertensive heart 
disease and hypertrophic cardiomyopathy. PLOS ONE. (2019) 14:e0221061. doi: 10.1371/journal.pone.0221061

94. Knott KD, Seraphim A, Augusto JB, Xue H, Chacko L, Aung N, et al. The prognostic significance of quantitative myocardial perfusion. Circulation. (2020) 141:1282-91. doi: 10.1161/CIRCULATIONAHA.119.044666

95. Swift AJ, Lu H, Uthoff J, Garg P, Cogliano M, Taylor J, et al. A machine learning cardiac magnetic resonance approach to extract disease features and automate pulmonary arterial hypertension diagnosis. Eur Heart J Cardiovasc Imaging. (2020). jeaa001. doi: 10.1093/ehjci/jeaa001. [Epub ahead of print].

96. Benjamin EJ, Virani SS, Callaway CW, Chamberlain AM, Chang AR, Cheng S, et al. Heart disease and stroke statistics-2018 update: a report from the American heart association. Circulation. (2018) 137:e6792. doi: 10.1161/CIR.0000000000000573

97. Min JK, Baskaran L. Ahead of the curve. Arterioscler Thromb Vasc Biol. (2019) 39:531-2. doi: 10.1161/ATVBAHA.119.312433

98. Leopold JA, Maron BA. Precision medicine in pulmonary arterial hypertension. Circ Res. (2019) 124:8323. doi: 10.1161/CIRCRESAHA.119.314757

99. Wu KC. A CURE for what ails in cardiac resynchronization therapy: defibrillator or pacemaker only? JACC Cardiovasc Imaging. (2020) 13:9379. doi: 10.1016/j.jcmg.2019.11.013

100. Kagiyama N, Shrestha S, Cho JS, Khalil M, Singh Y, Challa A, et al. A low-cost texture-based pipeline for predicting myocardial tissue remodeling and fibrosis using cardiac ultrasound. EBioMedicine. (2020) 54:102726. doi: 10.1016/j.ebiom.2020.102726

101. Krittanawong C, Johnson KW, Tang WW. How artificial intelligence could redefine clinical trials in cardiovascular medicine: lessons learned from oncology. Per Med. (2019) 16:83-8. doi: 10.2217/pme-2 018-0130

102. Bowman L, Baras A, Bombien R, Califf RM, Chen Z, Gale CP, et al. Understanding the use of observational and randomized data in cardiovascular medicine. Eur Heart J. (2020) 41:25718. doi: 10.1093/eurheartj/ehaa020

103. El Saddik A. Digital twins: the convergence of multimedia technologies. IEEE Multimedia. (2018) 25:87-92. doi: 10.1109/MMUL.2018.023121167

104. Maurovich-Horvat P. 2 steps forward, 1 step back. JACC Cardiovasc Imaging. (2019) 12(7 Pt 2):1377-8. doi: 10.1016/j.jcmg.2018.07.031

105. Bhavnani SP, Narula J, Sengupta PP. Mobile technology and the digitization of healthcare. Eur Heart J. (2016) 37:1428-38. doi: 10.1093/eurheartj/ ehv770

106. Bhavnani SP, Sola S, Adams D, Venkateshvaran A, Dash PK, Sengupta PP. A randomized trial of pocket-echocardiography integrated mobile health device assessments in modern structural heart disease clinics. JACC Cardiovasc Imaging. (2018) 11:546-57. doi: 10.1016/j.jcmg.2017.06.019

107. Singh S, Bansal M, Maheshwari P, Adams D, Sengupta SP, Price R, et al. American society of echocardiography: remote echocardiography with webbased assessments for referrals at a distance (ASE-REWARD) study. J Am Soc Echocardiogr. (2013) 26:221-33. doi: 10.1016/j.echo.2012.12.012

Conflict of Interest: PS was a consultant for HeartSciences and Ultromics.

The remaining authors declare that the research was conducted in the absence of any commercial or financial relationships that could be construed as a potential conflict of interest.

Copyright $\odot 2020$ Seetharam, Brito, Farjo and Sengupta. This is an open-access article distributed under the terms of the Creative Commons Attribution License (CC $B Y)$. The use, distribution or reproduction in other forums is permitted, provided the original author(s) and the copyright owner(s) are credited and that the original publication in this journal is cited, in accordance with accepted academic practice. No use, distribution or reproduction is permitted which does not comply with these terms. 